\section{Ironing out a yellow stripe}

Iron presents a quandary in biology. Its chemical properties have made it an essential metal for life, forming a vital part of cytochromes, globins and various redox enzymes. However, in its inorganic state it is relatively insoluble, resulting in the development of specialized systems for iron uptake and transport around living organisms. Iron is mainly taken up into the biosphere through the roots of plants, and in the 18 January issue of Nature, Elsbeth Walker and colleagues report the identification of a protein that is responsible for moving iron across this barrier in the world's major crop plants.

One strategy adopted by plants to absorb iron is to extrude from their roots small organic compounds called phytosiderophores. These bind tightly to the sparingly soluble iron in the soil, and are then reabsorbed into the roots, taking their chelated iron with them. Walker and colleagues have discovered the transporter for phytosiderophores back into the root in the classic mutant of maize known as yellow stripe 1 ( $y$ s1).

Yellow stripe 1 was first identified in 1929 by George W. Beadle, through its distinctive phenotype in which iron deficiency leads to incomplete pigmentation of leaves and alternating yellow and green stripes running their length (bottom in the figure, under a wild-type leaf). By the early 1990s it was known that $y s 1$ plants are defective in iron-phytosiderophore uptake, making the YS1 gene product a good candidate for the elusive phytosiderophore transporter.

To identify the YS1 gene, Walker's group used the Ac transposon to produce a library of mutant plants, one of whose disruptions proved allelic with $y s 1$. The $A c$-disrupted gene was then sequenced and its identity as yellow stripe confirmed by sequencing the allele from three separate $y s 1$ mutants.

The gene encodes a protein that contains twelve putative transmembrane regions, but is this really a phytosiderophore transporter? As a test, the YS1 gene was introduced into a mutant yeast (Saccharomyces cerevisiae) strain defective in extracting iron from the surrounding medium. This did not, on its own, rescue the yeast phenotype unless maize phytosiderophores were added to the growth medium.

At least one mystery remains. Maize's YS1 shows similarity to genes of unknown function from species throughout the plant kingdom monocots, dicots, gymnosperms and mosses many of which do not use phytosiderophores to absorb iron. Among those lacking phy-

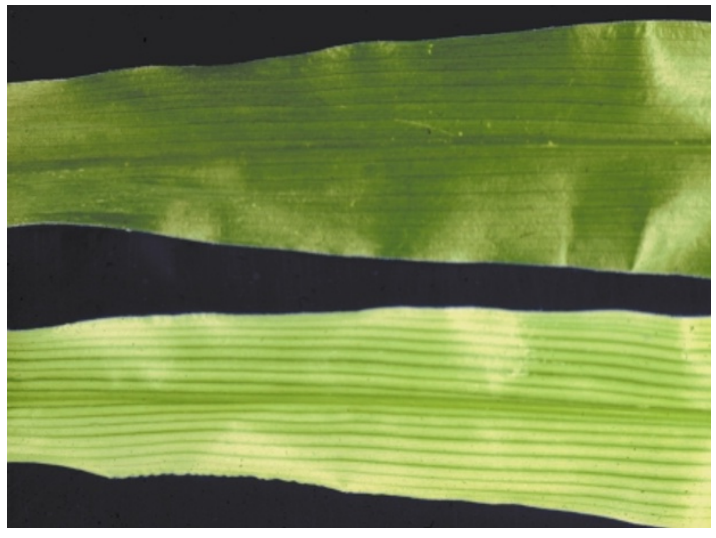

tosiderophores is Arabidopsis thaliana, but its recently sequenced genome contains no fewer than eight homologues of YS1. Perhaps these cousins are involved in the transport of iron within plants; for example by acting as a receptor for nicotianamine, an iron chelator found in all plants, which has been proposed to aid the longrange movement of iron through phloem tubes.

Iron deficiency is a leading nutritional disorder in the developing world, and this study brings us one step closer to staple crop varieties better able to accumulate bioavailable iron. Coming so close on the heels of the completion of the Arabidopsis genome sequence it also highlights the valuable genetic heritage of other, intensely studied plants. After all, Barbara McClintock discovered transposons, on which this and many other molecular biology studies are reliant, from work done entirely in maize.

Christopher Surridge Senior Editor, Nature

(2) References and links

ORIGINAL RESEARCH PAPER Curie, C. et al. Maize yellow stripe 1 encodes a membrane protein directly involved in $\mathrm{Fe}$ (III) uptake. Nature 409, 346-349 (2001)

\title{
On track with kinesin
}

Like a miniature railway, conventional kinesin can transport molecular cargoes over long distances. It does this without dissociating from its microtubule rails - a property known as processivity. But how does kinesin stay on track? In the Journal of Cell Biology, Ronald Vale and colleagues describe two studies aimed at finding out.

Conventional kinesin is a dimer, with two catalytic motor domains connected through a stalk to the cargo-binding carboxy-terminal tail. Each motor head is joined to the stalk by a flexible 'neck' region consisting of two parts - a 'neck linker' that interacts with the motor and an adjacent 'neck coiled-coil'. The neck linker drives the characteristic 'stepping' movement of the two kinesin heads, ensuring both heads do not dissociate simultaneously.

This mechanism relies on a conformational change, and there are two theories for how this might occur - that the neck linker is 'unzippered' from the motor, or that there is partial unwinding of the neck coiled-coil. Tomishige and Vale have now tried to distinguish these possibilities by manipulating movement of the neck region using disulphide crosslinking of cysteine residues engineered into recombinant kinesin motors.

Using this system to immobilize the neck linker, the authors found that kinesin no longer moved in only one direction. But crosslinking to permit limited movement allowed biased unidirectional diffusion of the kinesin. This indicates that partial movement of the neck linker is enough to determine directionality, but that full movement is required for active, processive movement. In support of the argument that conformational changes in the neck linker are needed for processivity, immobilization of the neck coiled-coiled region had very little effect on motor activity.

So what does this region do? Tomishige and Vale noticed that the extent of processivity — or 'run length' - was decreased by up to $50 \%$ when the neck coiled-coil was immobilized. In the second study, Thorn et al. investigated this further by adding positive charge to the neck coiled-coil to generate 'ultra-processive' kinesin mutants. The gain in processivity was diminished by high salt concentrations or by cleaving off the negative carboxyl terminus of the microtubule protein tubulin, indicating that there might be an electrostatic interaction between this region of tubulin and the neck coiled-coil. This interaction is probably weak, however, as it was abolished by adding relatively low loads to the kinesin in an optical-trap assay.

The current train of thought, then, is that the neck coiled-coil tethers kinesin near the microtubule surface, whereas the neck linker is involved in the conformational change behind processive movement.

Alison Mitchell

\section{(1) References and links}

ORIGINAL RESEARCH PAPERS Tomishige, M. \& Vale, R. D. Controlling kinesin by reversible disulfide cross-linking: identifying the motility-producing conformational change. J. Cell Biol. 151, 1081-1092 (2000) IThorn, K. S., Ubersax J. A. \& Vale, R. D. Engineering the processive run length of the kinesin Vale, R. D. Engineering the processive run lengt
motor. J. Cell Biol. 151, 1093-1100 (2000) WEB SITE The kinesin home page 